\title{
AVANTAGES DE LA CONFORMITÉ ET DE LA GESTION DES RISQUES
}

\section{ARTICLE ORIGINAL}

SILVA, Lilian Reis da ${ }^{1}$

SILVA, Lilian Reis da. Avantages de la conformité et de la gestion des risques. Revista Científica Multidisciplinar Núcleo do Conhecimento. Année. 06, Ed. 12, vol. 04, p. 123 à 147. Décembre 2021. ISSN : 2448-0959, Lien d'accès: https://www.nucleodoconhecimento.com.br/administration-des-affaires/avantagesde-la-conformite, DOI: 10.32749/nucleodoconhecimento.com.br/administration-desaffaires/avantages-de-la-conformite

\section{RÉSUMÉ}

La conformité est un programme qui vise à protéger les organisations contre la fraude financière, la corruption, le comportement et/ou l'inconduite des employés qui leur sont liés, empêchant ainsi leur bonne réputation et leur solidité financière d'être ébranlées. II s'agit d'un outil dont le but est d'établir des règles, des normes et des lignes directrices pour les processus internes au sein des organisations. Il a été développé aux États-Unis en 1970, et ses pratiques ont été transformées en un institut juridique, grâce à la promulgation de la loi pionnière contre la corruption FCPA (Foreign Corrupt Practices Act), motivée par l'affaire du Watergate, qui impliquait le président de l'époque, Richard Nixon, et des membres de son administration, en payant des potsde-vin dans des enquêtes en faveur de sa réélection. Dans ce contexte, cet article a pour question principale: comment les avantages offerts par le programme de conformité et de gestion des risques collaborent-ils pour réduire les risques de fraude, d'illicite et de corruption au sein des organisations ? L'objectif de cette étude était de présenter les avantages offerts par l'adoption du programme de conformité et de

\footnotetext{
${ }^{1}$ Postgraduate en Gestion d'Entreprise, économiste et technique comptable.

RC: 103917

Disponible en: https://www.nucleodoconhecimento.com.br/administration-desaffaires/avantages-de-la-conformite
} 
gestion des risques dans les entreprises publiques et privées, dans leurs processus internes et leurs relations avec leur secteur d'activité, et comment elles collaborent pour atténuer la fraude. La méthodologie a été adoptée comme une recherche bibliographique, et il s'agissait des avantages causés par les programmes de prévention de la fraude. II a été constaté que les programmes de conformité et de gestion des risques apportent des avantages efficaces en matière de protection contre les risques, atténuant la fraude et la corruption, combinés à de nouvelles solutions de gouvernance des Technologies de l'Information ( $\mathrm{TI})$, telles que la Enterprise Governance, Risk Management and Compliance (EGRC).

Mots-clés: Fraude au sein des organisations, Prévention des risques de corruption, Conformité.

\section{INTRODUCTION}

Discuter des avantages de la conformité et de la gestion des risques implique de présenter des concepts sur ce qu'est un programme de conformité et quels sont les risques que les entreprises courent lorsqu'elles n'adoptent pas ce type de programme, compte tenu des différents types de fraude, y compris la corruption, le blanchiment d'argent, les manipulations avec des informations internes et/ou lorsque les employés commettent des actions qui peuvent nuire à l'image d'une organisation, peut même causer des pertes financières.

Selon Michelotti (2020, p. 6), l'adoption d'un programme de conformité et de gestion des risques assure la transparence des organisations, démontrant l'existence d'une conduite éthique; en outre, il collabore avec les pratiques de bonne gouvernance d'entreprise, qui est «l'ensemble des processus par lesquels les entreprises sont gérées ».

Selon Sobreira Filho, Leite e Martins (2019, p. 23), « le programme de conformité est un outil d'entreprise dont la méthodologie de mise en œuvre est basée sur l'éthique des affaires et l'intégrité comme sa plus grande valeur ».

RC: 103917

Disponible en: https://www.nucleodoconhecimento.com.br/administration-desaffaires/avantages-de-la-conformite 
Le terme conformité (aussi appelée compliance), nédu verbe anglais « to comply » est défini, résumé par Coelho et Santos Jr (2021, p. 8) comme " satisfaire les impositions de l'ordre juridique ou de l'ordre interne de l'entreprise » (griffons de l'original). A ce concept, Gonsales (2016) ajoute que " si au début, la conformité signifiait "suivre les règles ", d'après les grandes enquêtes brésiliennes, cela signifiait aussi « suivre la loi anti-corruption ».

Il convient de noter que la conformité s'applique aux entreprises publiques ou privées, de différentes tailles et segments, situées dans tous les pays, aux organisations qui ont le désir d'agir avec éthique, morale et dans la promotion du développement humain, social, économique et financier.

Cet article a été développé comme une recherche bibliographique, pour laquelle la question suivante a été définie: comment les avantages offerts par le programme de conformité et de gestion des risques collaborent-ils pour réduire les risques de fraude, d'illicite et de corruption au sein des organisations? À son tour, l'objectif général était de présenter les avantages offerts par l'adoption du programme de conformité et de gestion des risques dans les entreprises publiques et privées, dans leurs processus internes et leurs relations avec leur secteur d'activité, et comment elles collaborent pour atténuer la fraude.

\section{CONFORMITÉ}

\subsection{CONCEPT}

Pour qu'une entreprise soit considérée comme fiable, inspirant la sécurité dans son segment d'activité et devant la société, il est important qu'elle ait des valeurs basées sur la morale, l'éthique et la justice. Elle doit agir avec transparence, ce qui lui permet de construire sa crédibilité, et aussi attirer des talents humains qui renforcent sa capacité d'action, en visant sa croissance (JORGE et TOMAZ, 2018). 
Dans cette perspective, pour qu'une organisation agisse avec éthique, bons principes et compétitivité, la lutte contre la fraude est un enjeu fondamental, inhibant tout type d'illicite, par l'adoption de pratiques et de processus pour protéger ses valeurs et ses objectifs, en adoptant un programme de conformité.

La conformité suppose «le accession et le respect des normes et réglementations ». Selon Antonik apud Jorge et Tomaz (2018, p. 108), conformité (aussi appelée compliance) vient de l'anglais, « to comply », qui signifie « règles, instructions, normes, directives ou simplement répondre à une commande ».

Coelho et Santos Júnior (2021, p. 8) soulignent que "l'objectif des normes de conformité est de se concentrer sur le résultat à atteindre, c'est-à-dire d'éviter les risques découlant de la commission d'un comportement personnel ou organisationnel considéré comme illégal ou incompatible avec les principes, les missions, la vision ou les objectifs d'une entreprise ».

Au fil du temps, entre 1907 et 1977, des lois sont apparues applicables aux États-Unis avec une portée internationale également, pour contrôler et punir les citoyens, les entreprises et les agences gouvernementales qui se livrent à des actions de corruption.

Selon Nascimento (2018), la conformité a été créée aux États-Unis, vers les années 1970, élevée au rang d'institut juridique à partir de la promulgation de la loi pionnière pour lutter contre la corruption ou - Foreign Corrupt Practices Act (FCPA). Une telle législation a été élaborée et mise en œuvre après l'affaire du Watergate, qui a abouti à la démission du président des États-Unis de l'époque, Richard Nixon.

Furtado et Rocha (2015, apud MICHELOTTI, 2020, p. 7-8) rapportent que dans la période précédant la loi FCPA, les pratiques du capitalisme dans l'ouverture de nouveaux marchés comprenaient le paiement de pots-de-vin, « considéré comme une étape nécessaire et naturelle dans l'environnement des entreprises. II y avait la possibilité la plus claire que le pot-de-vin soit inscrit comme dépenses dans le bilan. 
Et la corruption pour obtenir des contrats ou des concessions dans ces pays faisait partie des règles du jeu. »

Puisque la corruption est un problème mondial, et implique - dès le début - les gouvernements, les personnes, les institutions publiques et/ou privées, Ubirajara Costódio Filho, mentionné par Nascimento (2021) affirme que c'est quelque chose qui nuit à toutes les sphères sociales, en ce qui concerne l'environnement, la libre concurrence, les finances publiques, le commerce international, démocratie et préceptes républicains.

Toujours sur la corruption, Nascimento (2021, n.p.) rapporte également Eduardo Cambi, pour souligner que:

a corrupção atrasa o desenvolvimento econômico e social. Restringe a vontade soberana do povo. Apropria a coisa pública para a realização de interesses privados. Gera promiscuidade entre o poder público e o poder econômico. Concentra renda. Ressalta privilégios e desigualdades. Impede a universalização de políticas públicas e a concretização de direitos fundamentais. Destrói a cidadania e enfraquece a democracia.

Le Foreign Corrupt Practices Act (FCPA), ou Loi Américaine Contre les Pratiques de Corruption, avait pour objectif de punir les actions frauduleuses des entreprises offrant/payant des pots-de-vin à des agents publics, dans le but de développer leurs activités dans le monde. Dès l'adoption des pratiques prescrites par la loi FCPA, toutes les organisations sont tenues de suivre des réglementations internes et externes fondées sur les questions éthiques et morales nécessaires aux relations commerciales et économiques avec leurs partenaires (NASCIMENTO, 2018).

\section{2 ÉMERGENCE D'UNE LÉGISLATION SUR LES PRATIQUES DE CONFORMITÉ}

Selon Nascimento (2018, p. 26), le mouvement mondial de lutte contre la corruption et la pratique enracinée de la corruption " a commencé avec le Foreign Corrupt Practices Act (FCPA), ou Loi Américaine Contre les Pratiques de Corruption ». Cet

RC: 103917

Disponible en: https://www.nucleodoconhecimento.com.br/administration-desaffaires/avantages-de-la-conformite 
auteur précise que « le degré de corruption dans un pays donné varie en fonction de la façon dont les multinationales y agissent ».

Parmi les taux de corruption vérifiés, Nascimento (2018, p. 26-27) rapporte la proportion d'entreprises dans le monde où la corruption illicite et le blanchiment d'argent persistent:

$23 \%$ das empresas na União Europeia e Europa Ocidental; $66 \%$ das empresas na América Latina; $64 \%$ das empresas na Ásia; 95\% das empresas na Europa Ocidental e da Ásia Central; 84\% das empresas do Oriente Médio e do Norte da África; $90 \%$ das empresas da África Subsaariana.

Cet auteur (NASCIMENTO, 2018, p. 26-27) affirme que la mondialisation économique " a engendré le crime mondial de corruption lié au blanchiment d'argent et à la corruption offshore ».

On observe donc que la littérature sur les programmes de conformité et de gestion des risques montre que, à un moment donné, plusieurs pays et leurs gouvernements ont décidé de faire face à des pratiques illégales - tant dans les institutions publiques que dans les organisations privées - qu'ils ont depuis lors été contraints d'adopter des mesures punitives et restrictives pour les citoyens et les pays qui commettent des fraudes, la corruption et le blanchiment d'argent, entre autres, aboutissant à des traités internationaux.

Les pays qui adoptent des programmes de prévention de la fraude sont signataires de l'Organisation de Coopération et de Développement Économiques (OCDE), notamment : Allemagne, Australie, Autriche, Belgique, Canada, Chili, Corée, Danemark, Slovénie, Espagne, États-Unis, Estonie, Finlande, France, Grèce, Hongrie, Irlande, Islande, Israël, Italie, Japon, Lettonie, Luxembourg, Mexique, Norvège, Nouvelle-Zélande, Pays-Bas, Pérou, Pologne, Portugal et Royaume-Uni (NASCIMENTO, 2021, s.p.).

Pour clarifier la façon dont ces pays ont choisi d'adopter de bonnes pratiques de gouvernance d'entreprise, il est important de faire émerger des institutions et des lois 
américaines qui ont permis l'adoption d'instituts de protection concernant les différents types d'infractions illicites contre les organisations, entraînant la promulgation de lois aux mêmes fins dans le monde entier.

En 1907, la Conférence de La Haye est créée, qui est « une cour internationale de justice pour enquêter sur les conflits internationaux ». Avec elle, une portée pour la fondation de la Banque des Règlements Internationaux (BIS) (Bank for International Settlements - BIS), visant à promouvoir « la coopération entre les banques centrales pour parvenir à une plus grande stabilité financière ". Cet objectif est considéré par les experts comme «les premières racines de la conformité » (NASCIMENTO, 2021, s.p.).

En avril 1948, l'Organisation européenne de coopération économique (OECE) est née au Portugal, visant la coopération économique entre les pays européens. Sa composition initiale comptait 18 pays, à souligner: Autriche, Belgique, Danemark, France, Grèce, Hollande, Irlande, Islande, Italie, Luxembourg, Norvège, Portugal, Royaume-Uni, Suède, Suisse, Turquie et Allemagne de l'Ouest.

En 1948 également, l'Organisation des États américains (OEA) (Organization of American States - OAS) repose sur quatre piliers: « démocratie, droits de l'homme, sécurité et développement ». (NASCIMENTO, 2021, s.p.).

Toujours Nascimento (2021, s.p.), souligne ce que Sanches et Renee (2017, p. 18) défendent à propos de l'OEA : « cette convention vise à promouvoir et à renforcer les mécanismes nécessaires pour aider à prévenir, détecter et punir la corruption dans l'exercice des fonctions publiques, ainsi que les actes de corruption spécifiquement liés à son exercice ».

En décembre 1960, les membres de l'OECE ont signé une nouvelle convention avec les États-Unis et le Canada qui a créé l'Organisation pour de coopération et de développement économiques (OCDE), remplaçant I'OECE. Ainsi, en 1961, arrive I'Organisation pour de coopération et de développement économique (OCDE) (Organisation for Economic Co-operation and Development (OECD), succédant à

RC: 103917

Disponible en: https://www.nucleodoconhecimento.com.br/administration-desaffaires/avantages-de-la-conformite 
l'Organisation Européenne de Coopération Économique, dont les objectifs étaient de " couvrir le développement économique et la stabilité financière des pays membres " (NASCIMENTO, 2021, s.p.).

En 1975, le Comité de Bâle sur le contrôle bancaire (BCBS) (Basel Committee on Banking Supervision - BCBS), a été fondé, dont l'objectif était de " réglementer et superviser les meilleures pratiques financières ". En 1998, elle a promu une uniformité des règles applicables aux institutions financières, visant à maintenir les bonnes pratiques de gouvernance d'entreprise (NASCIMENTO, 2021, s.p.).

En 1977, face au scandale du Watergate, la loi américaine contre les pratiques de corruption à l'étranger (FCPA) a été développée, étant la 1ère. loi anti-corruption, publiée plus tard en 1988 et 1998 . Selon Nascimento (2021, s.p.), dans le but d'«adopter des sanctions civiles, pénales et administratives aux personnes physiques et morales contre les pratiques de corruption, le Foreign Corrupt Practices Act (FCPA), ou Loi Américaine Contre les Pratiques de Corruption» (NASCIMENTO, 2021, s.p.).

II est à noter que la FCPA décrit comme " crime le paiement de pots-de-vin directement ou indirectement, les paiements aux fonctionnaires étrangers, aux partis politiques, le paiement non enregistré de manière concise, il doit y avoir des livres de registres comptables » (NASCIMENTO, 2021, s.p.).

Ces enquêtes ont été abordées par la Fondation Getúlio Vargas (FGV) (2021, s.p.), signalant l'émergence de la Securities and Exchange Commission (SEC), qui est " une organisation administrative chargée de superviser et de lutter contre les pratiques de corruption dans les transactions internationales ».

La SEC «a trouvé des pratiques de paiements illégaux à des agents publics par une moyenne de 400 entreprises privées et publiques américaines, ainsi que des sociétés étrangères ". Dans une telle déclaration, la SEC a constaté qu'«ensemble, ils ont versé plus de 300 millions de dollars en pots-de-vin à des représentants de gouvernements étrangers » (FGV, 2021, s.p.). 
En 1989, pour une action conjointe avec l'OCDE, le Groupe d'action financière (GAFI) (Financial Action Task Force-FATF), a été créé, visant à élaborer des politiques et à réformer la législation et la réglementation, sur la base du droit international, visant à lutter contre le blanchiment de capitaux et le financement du terrorisme. Le GAFI agit également dans la composition des mécanismes d'évaluation périodique d'autres pays (NASCIMENTO, 2021, s.p.).

Dans la pratique, le texte de l'OEA a amené la Convention interaméricaine contre la corruption, signée en 1996, visant à « protéger les intérêts des États-Unis en termes de terrorisme et de corruption ».

En 2002, la loi Sarbanes Oxley (SOX) (Sarbanes-Oxley Act - SARBOX) a été mise en œuvre pour mieux promouvoir " la gouvernance d'entreprise, la surveillance et la prévention des pratiques préjudiciables, l'augmentation des contrôles et de la transparence ». Avec la création de l'Organe de supervision du travail des auditeurs indépendants, il a également institué des audits indépendants pour les cas suspects, renforçant " la responsabilité en matière de fraude d'entreprise ou criminelle, augmentant le niveau de divulgation d'informations financières, augmentant les sanctions pour les crimes en col blanc (NASCIMENTO, 2021, s.p.).

En 2010, le UK Bribery Act (BA) a émergé au Royaume-Uni, caractérisant quatre crimes: "corruption active de particuliers publics et privés, corruption passive de particuliers publics et privés, corruption d'agents publics étrangers, incapacité des entreprises à prévenir la corruption » (NASCIMENTO, 2021, s.p.).

À leur tour, Madruga et Belloto ont élaboré un guide présentant 10 piliers qui, une fois adoptés, permettent la mise en œuvre d'un programme de conformité efficace (NASCIMENTO, 2018, p. 8-9) :

1. Compromisso de todos os níveis da gerência e política anticorrupção claramente articulada; 2 . Código de conduta e política e procedimentos de compliance; 3. Fiscalização interna do programa, autonomia da área de compliance e recursos adequados; 4. Avaliação dos riscos da empresa; 5. Treinamentos e aconselhamento contínuo; 6. Incentivos e medidas disciplinares; 7. Investigações (due diligence) de terceiros e de pagamentos; 8.

RC: 103917

Disponible en: https://www.nucleodoconhecimento.com.br/administration-desaffaires/avantages-de-la-conformite 
Sistema interno de denúncias (possibilitando denúncias confidenciais) e sistema de investigações internas; 9. Melhoria contínua: testes e revisões periódicas do programa de compliance; 10. Fusões e Aquisições: Investigações (due diligence) pré-fusões e aquisições e integrações pós-aquisições e fusões.

En ce qui concerne le Brésil, et malgré la promulgation de la loi $n^{\circ} 12.846 / 13$, dont l'objet est l'adoption de mesures juridiques et de procédures internes à suivre par les organisations à travers l'adoption d'un code de conduite pour lutter contre la corruption, l'entrée dans l'OCDE n'a pas encore été officialisée par cet organe, compte tenu des cas constants de corruption, la fraude et le blanchiment d'argent qui surviennent ici (DURÃES et RIBEIRO, 2020).

\subsection{LES PRATIQUES FRAUDULEUSES ET LES PERTES CAUSÉES PAR DES TIERS À DES ORGANISATIONS}

Lorsque des personnes liées à une organisation donnée adoptent des comportements qui nuisent à l'image de l'entreprise, leurs actions erronées ont des répercussions négatives sur elle, ainsi que sur l'ensemble de la société. Il existe de nombreux cas de comportement répréhensible qui ébranlent non seulement l'image, mais entraînent également des pertes, des amendes et parfois, la faillite.

En ce qui concerne les différents types de fraude au sein des organisations et institutions gouvernementales, tels que la fuite d'informations confidentielles, la corruption passive/ active, le blanchiment d'argent, entre autres, Gonsales (2016) affirme que toutes les études liées à une telle fraude soutiennent que dans chaque entreprise, elles se produisent. Ainsi, plus votre leader croit que rien de mal ne se passe, plus votre organisation devient vulnérable.

Au Brésil, il existe des pratiques frauduleuses connues dans toute la société, telles que "falsifier la licence d'un étudiant ou voler des lignes de télévision par câble ", mentionnées par Condé et al. (2015, p. 95); ce sont des actes qui consistent à démontrer la mauvaise foi des uns et leur intention claire de tromper les autres. Malheureusement, ce sont des actions pratiquées par beaucoup de gens, mais qui au fil du temps sont devenues si courantes qu'elles finissent par être acceptées dans la

RC: 103917

Disponible en: https://www.nucleodoconhecimento.com.br/administration-desaffaires/avantages-de-la-conformite 
culture brésilienne. Ce sont des actes qui démontrent une mentalité et un comportement contraires à l'éthique et illégaux; cependant, ils sont considérés comme non nocifs pour la société, ce qui est faux.

Par rapport aux actions ci-dessus, Condé et al. (2015, p. 95) suggère que si des pratiques contraires à l'éthique, frauduleuses et corrompues étaient menées par des cadres supérieurs d'une organisation, elles seraient considérées comme nuisibles et inacceptables par la société.

À cet égard, Cossenzo (2015, p. 35) rapporte qu'Oliveira (2012, p. 115) définit qu'« une fraude financière est commise à partir d'actes volontaires de son agent, avec l'intention claire de nuire financièrement à autrui ». Cet auteur poursuit en expliquant que la fraude peut être (i) interne - si elle est commise par des employés contre l'organisation; (ii) externe - s'il est commis par des tiers non membres de la société; (iii) mixte: si des actions frauduleuses sont menées par des agents internes et externes.

Selon cette explication, il convient de souligner un concept lié à la fraude financière, considéré comme " quelque chose d'inhérent à l'entreprise » de ce type d'institution, se classant comme des risques opérationnels liés à la fois à ce segment et « aux personnes, aux processus et à la technologie »(COSSENZO, 2015, p. 35).

II convient de préciser que la conformité couvre tous les types de fraude pouvant survenir dans différents secteurs d'activité, c'est-à-dire que la conformité cherche à atténuer les risques pouvant être causés par une conduite personnelle ou organisationnelle (qu'ils soient dans le secteur financier ou non), s'ils n'agissent pas conformément à la conduite éthique et à la politique de l'entreprise.

RC: 103917

Disponible en: https://www.nucleodoconhecimento.com.br/administration-desaffaires/avantages-de-la-conformite 


\section{4 ÉVÉNEMENTS PRÉJUDICIABLES À LA RÉPUTATION DES ENTREPRISES AMÉRICAINES}

Selon Haussen (2021, p. 9), « la réputation d'une organisation peut être fortement ébranlée par un comportement déconnecté découlant de l'absence de mise en œuvre d'un programme de conformité ",comme en témoignent les cas de corruption qui se sont produits aux États-Unis, qui ont stumené dans les médias au fil des ans, y compris l'affaire du Watergate - qui a conduit à la promulgation de la loi FCPA, en plus des autres mentionnés tout au long de cet article.

Parmi les nombreux cas d'actions préjudiciables à l'image des grandes entreprises américaines, compromettant leur crédibilité, seront mis en évidence ici certains qui ont attiré beaucoup d'attention dans les médias, comme le démontrent certains rapports situés dans les médias électroniques. Ce sont des histoires qui rapportent comment le manque de mise en œuvre d'un programme de conformité pour guider les processus et les personnes au sein d'une organisation finit par permettre à certains employés de ternir l'image et la réputation d'entreprises mondialement connues, avec des actions répréhensibles.

Le premier rapport de Vaz (2012) publié par Exame Magazine apporte un cas qui s'est produit dans le géant Apple, dont le texte intégral est présenté ici.

A Empresa Apple foi acusada pelo Senado nos Estados Unidos de usar filiais no exterior para evitar o pagamento de bilhões de dólares em impostos. "A Apple não apenas transferiu seus lucros para um paraíso fiscal estrangeiro, mas criou um 'Santo Graal' de evasão fiscal ao montar entidades no exterior para driblar o fisco", disse o senador democrata Carl Levin.

Uma reportagem do new York Times revelou como a Apple tem feito para driblar bilhões de dólares em impostos nas suas operações nos Estados e em outros países. Segundo Martin A. Sullivan, um ex-economista do Tesouro americano, sem as táticas utilizadas para a gestão dos impostos, a empresa liderada por Tim Cook teria deixado pelo menos mais 2,4 bilhões de dólares em taxas federais nos EUA no ano passado. A empresa declarou ter pagado 3,3 bilhões de dólares em impostos ao redor do mundo em 2011 sobre os lucros de 34,2 bilhões de dólares, uma taxa de $9,8 \%$. A companhia declara que $30 \%$ dos lucros vem dos Estados, caso o percentual pulasse para $50 \%$, os

RC: 103917

Disponible en: https://www.nucleodoconhecimento.com.br/administration-desaffaires/avantages-de-la-conformite 
pagamentos saltariam em 2,4 bilhões de dólares. "É razoável esperar que $70 \%$ dos lucros viriam dos Estados Unidos”, calcula.

Figure 1 : Symbole Apple

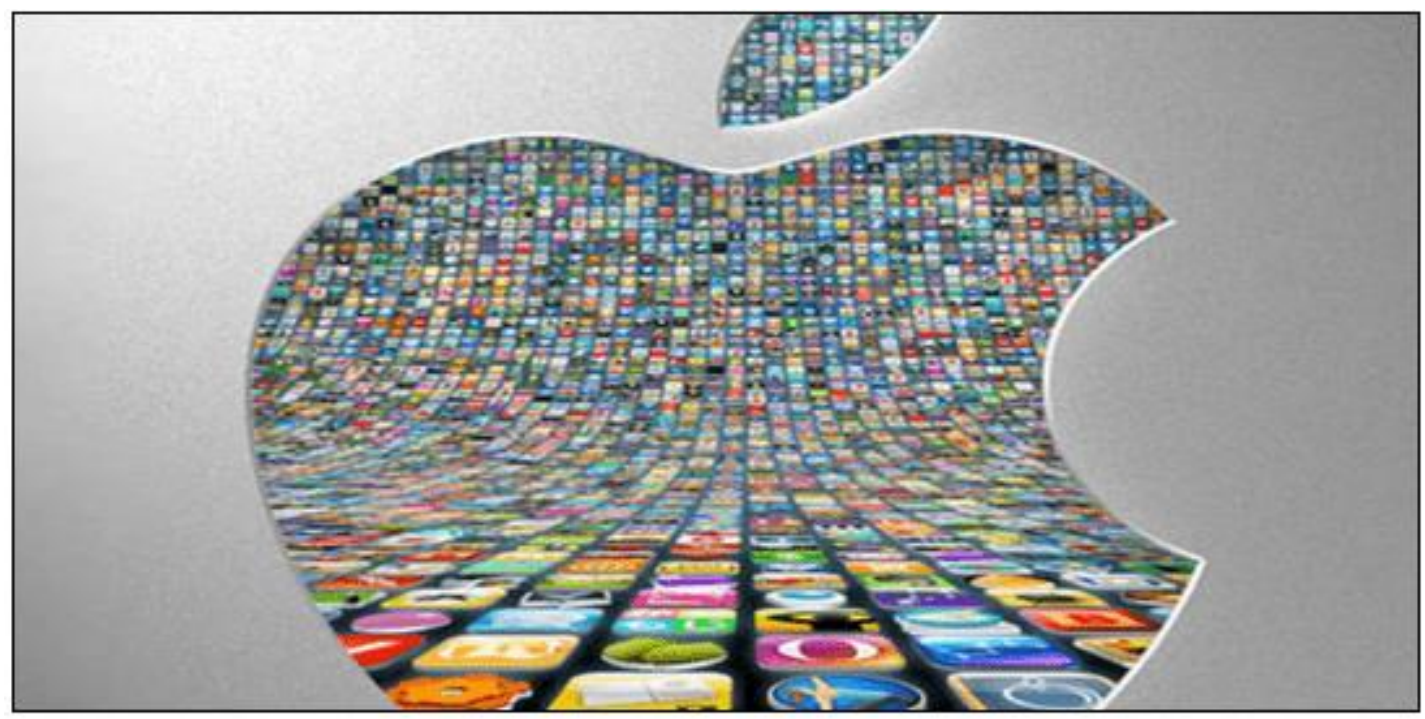

Source: Vaz (2012)

En ce qui concerne les causes possibles qui pourraient compromettre la crédibilité des organisations, Souza; Maciel-Lima et Lupi (2018, p. 13) se réfèrent à Gonsales (2016), pour expliquer que:

o valor de uma empresa está ligado à sua reputação e no caso de uma empresa ser citada em jornais e meios de comunicação, ainda que seja um ato ilícito isolado, acometido por um funcionário, a empresa sofrerá abalo a reputação e passa a ser conhecida no mercado como uma empresa corrupta.

Le deuxième rapport fait référence à deux situations différentes, qui se sont produites dans deux unités différentes dans les restaurants nord-américains du réseau Taco Bell, fast food inspirée de la cuisine mexicaine, qui suit le modèle McDonalds.

Le premier est dans le rapport de melo (2014) publié par Exame Magazine, dont le texte suit ci-dessous.

Publicar uma foto de si mesmo fazendo xixi em um prato do Taco Bell, nos Estados Unidos, não custou ao atendente Cameron Jankowski apenas o emprego. Indignados, hackers divulgaram os dados pessoais do "brincalhão"

RC: 103917

Disponible en: https://www.nucleodoconhecimento.com.br/administration-desaffaires/avantages-de-la-conformite 
na internet, segundo o Huffington Post. Em 2012, Jankowski postou imagem no Twitter, mas disse que não chegou a servir a comida no restaurante. Ele chegou a deletar sua conta, mas acabou sem seu emprego na rede.

Figure 2: Panneau électronique Taco Bell

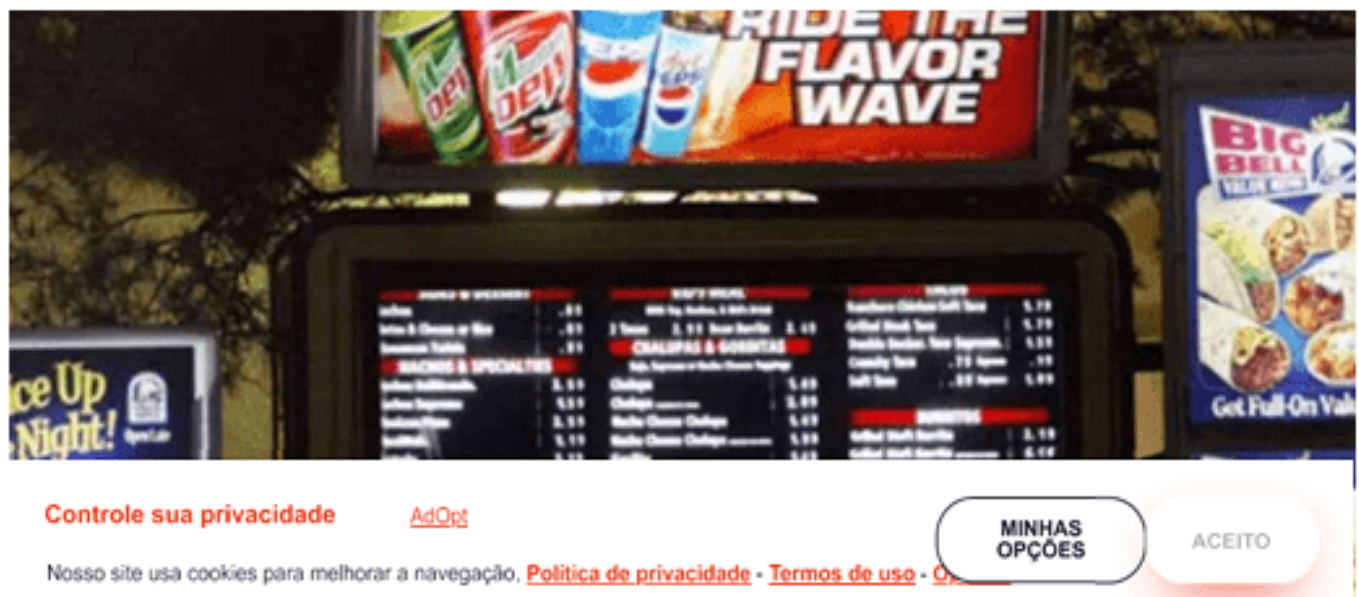

Source: Melo (2014)

En 2013, le même réseau aurait traversé un autre moment qui aurait nui à sa crédibilité. Le rapport est tiré du Magazine Veja On Line (2014), dont le texte suit dans son intégralité.

Uma imagem de um funcionário da rede de comida mexicana Taco Bell lambendo uma pilha de tortilhas foi postada na página da empresa no Facebook. A foto, de autoria do usuário Jj O'Brien Nolan, é seguida da legenda "isto com certeza diz muito sobre seus empregados, o tratamento da comida e o que eles postam na internet.

Apesar de inicialmente acreditar que a foto fosse uma brincadeira, a empresa demitiu o empregado. Além disso, em resposta, a Taco Bell divulgou um comunicado oficial, além de comentar a postagem de Nolan no Facebook. A empresa se defendeu afirmando que todas as unidades têm rígidos procedimentos de manuseamento e tolerância zero para violação dos alimentos.

RC: 103917

Disponible en: https://www.nucleodoconhecimento.com.br/administration-desaffaires/avantages-de-la-conformite 
Figure 3: Un employé de Taco Bell léchant un tas de tortillas

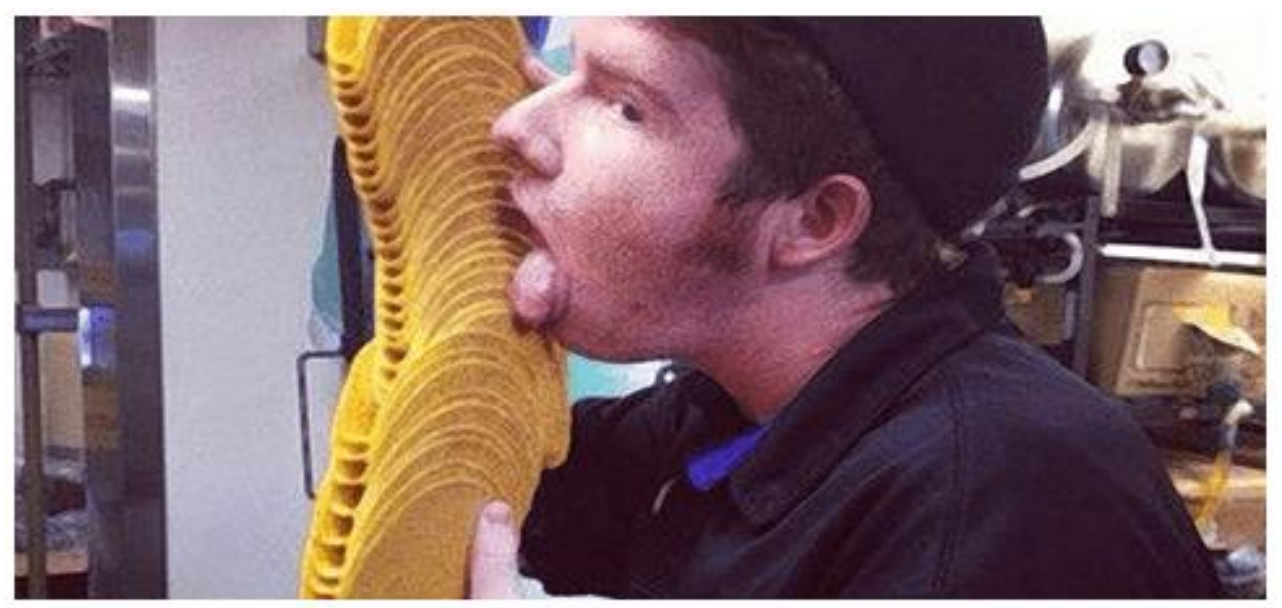

Source : Veja en ligne (2014)

Un troisième cas à mentionner a été publié dans un rapport de Melo (2014, publié par Exame Magazine, qui s'est produit dans l'un des restaurants du réseau KFC, en Amérique du Nord, dont le texte original est reproduit ici.

Ce fut le cas lorsqu'une ancienne employée de la chaîne de restaurants KFC aux États-Unis a publié une photo sur Facebook alors qu'elle léchait littéralement la purée de pommes de terre. Elle et son ami photographe ont été licenciés. Le réseau a dû se justifier en disant que la nourriture n'était pas servie. 
Figure 4: Employé de KFC léchant de la purée de pommes de terre
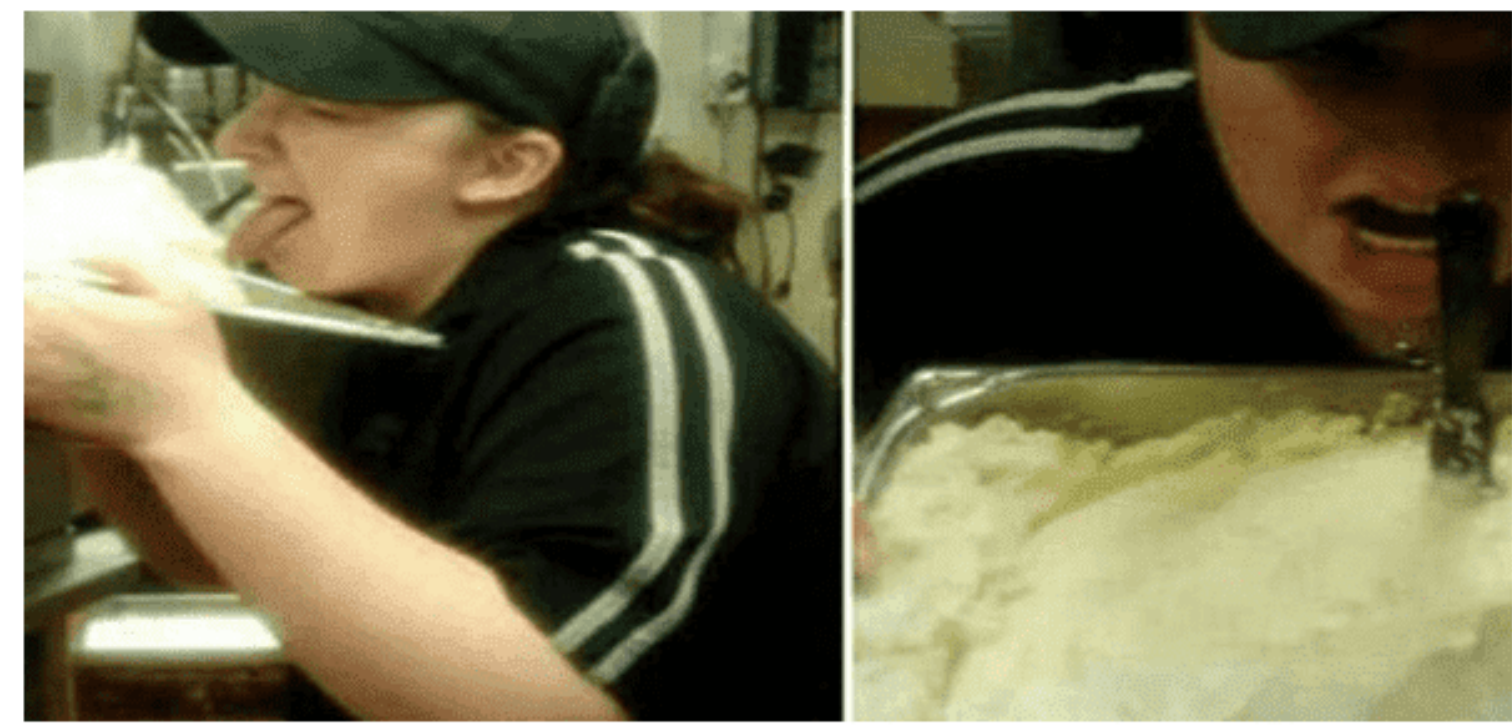

Source: Melo (2014)

Les cas de conduite contraire à l'éthique présentés reflètent l'importance de l'adoption de pratiques de conformité et de gestion des risques. Du point de vue de Coelho et Santos Júnior (2021, p. 26), « il ne suffit pas que l'entreprise ait la situation financière d'investir dans la publicité et le marketing pour dominer le marché ».

Ce qui est vérifié, dans la pratique, c'est que les organisations doivent se tenir à jour en permanence, à l'écoute des nouvelles pratiques de marketing, en plus d'adopter des stratégies qui leur permettent d'être compétitives dans leur segment d'activité, d'offrir de meilleurs services, des prix compétitifs et de donner des options de choix à leurs consommateurs cibles.

En ce sens, Coelho et Santos Júnior (2021, p. 27) soutiennent que « parmi les facteurs qui influencent le niveau de compétitivité des entreprises figurent: la capacité d'innovation, les produits et services de qualité, les différentiels commerciaux, la capacité de production et de livraison, la portée géographique, les actions de commercialisation; entre autres. 


\section{RISQUES DE CONFORMITÉ}

En omettant d'adopter des programmes de prévention et d'atténuation de la fraude dans leurs processus organisationnels, les entreprises sont sujettes à l'événement de différents événements illicites, puisque " la fraude se produit dans toutes les entreprises ». De plus, ils sont vulnérables à deux types de risques très dommageables: le bouleversement de leur réputation et les sanctions prévues par la nouvelle loi anti-corruption (GONSALES, 2016).

Le terme "Risque de Conformité » désigne les risques auxquels les entreprises sont soumises par des sanctions réglementaires, des pertes financières et/ou des pertes causées à leur image, des risques découlant du non-respect de la réglementation, des codes de conduite et de bonne conduite (COELHO et SANTOS JR, 2021, p. 8).

Selon Gonsales (2016), 43\% des entreprises brésiliennes n'ont pas encore adopté de programmes et de politiques de conformité pour leurs processus et leur protection, mais que bientôt toutes seront obligées de le faire, visant à protéger leur réputation, car « la valeur d'une entreprise est liée à sa réputation.

C'est une réalité qui a transformé l'environnement commercial et industriel national, découlant des enquêtes de Mensalão et de l'opération Lava Jato et par la suite, puisque le démantèlement de la fraude financière et de la corruption et du blanchiment d'argent prouvés démontrent la nécessité d'un nouvel environnement commercial, qui exige la transparence et l'intégrité dans la conduite des affaires. Les sanctions et les arrestations de cadres supérieurs et de hauts responsables gouvernementaux ont eu lieu à la lumière de la nouvelle loi anti-corruption instituée dans le pays (GONSALES, 2016).

Les données étudiées par PwC Brésil (2020, p. 4) montrent que le risque de conformité en matière de fraude et de crimes économiques les plus courants se produit avec des organisations à l'échelle nationale et internationale. Selon cette recherche, il a été constaté qu'au Brésil figurent parmi les trois types de fraude les plus courants: (1) les

RC: 103917

Disponible en: https://www.nucleodoconhecimento.com.br/administration-desaffaires/avantages-de-la-conformite 
pots-de-vin et la corruption, (2) la fraude comptable et (3) la fraude commise par le consommateur. Depuis que l'enquête a été menée en 2020, il a été démontré qu'au cours des deux dernières années (entre 2018 et 2019), les fraudes les plus courantes qui sont apparues dans les premières positions étaient: (a) les vols d'actifs, (b) la fraude dans les achats et (c) les pots-de-vin et la corruption.

À leur tour, selon PwC (2020, p. 4), les données comparatives du classement des fraudes qui apparaissent dans les premières positions dans le monde étaient: (1) la fraude commise par les consommateurs, (2) la cybercriminalité et (3) le vol d'actifs. Depuis que l'enquête a été menée en 2020, il a été démontré qu'au cours des deux dernières années (entre 2018 et 2019), les fraudes les plus courantes qui sont apparues dans les premières positions étaient: (a) la fraude commise par le consommateur, (b) la fraude comptable (c) la concurrence déloyale, (d) la fraude aux ressources humaines et (e) les pots-de-vin et la corruption.

La figure 5 montre les données dans les perspectives internes et externes. 
Figure 5 - Les types de crimes les plus courants

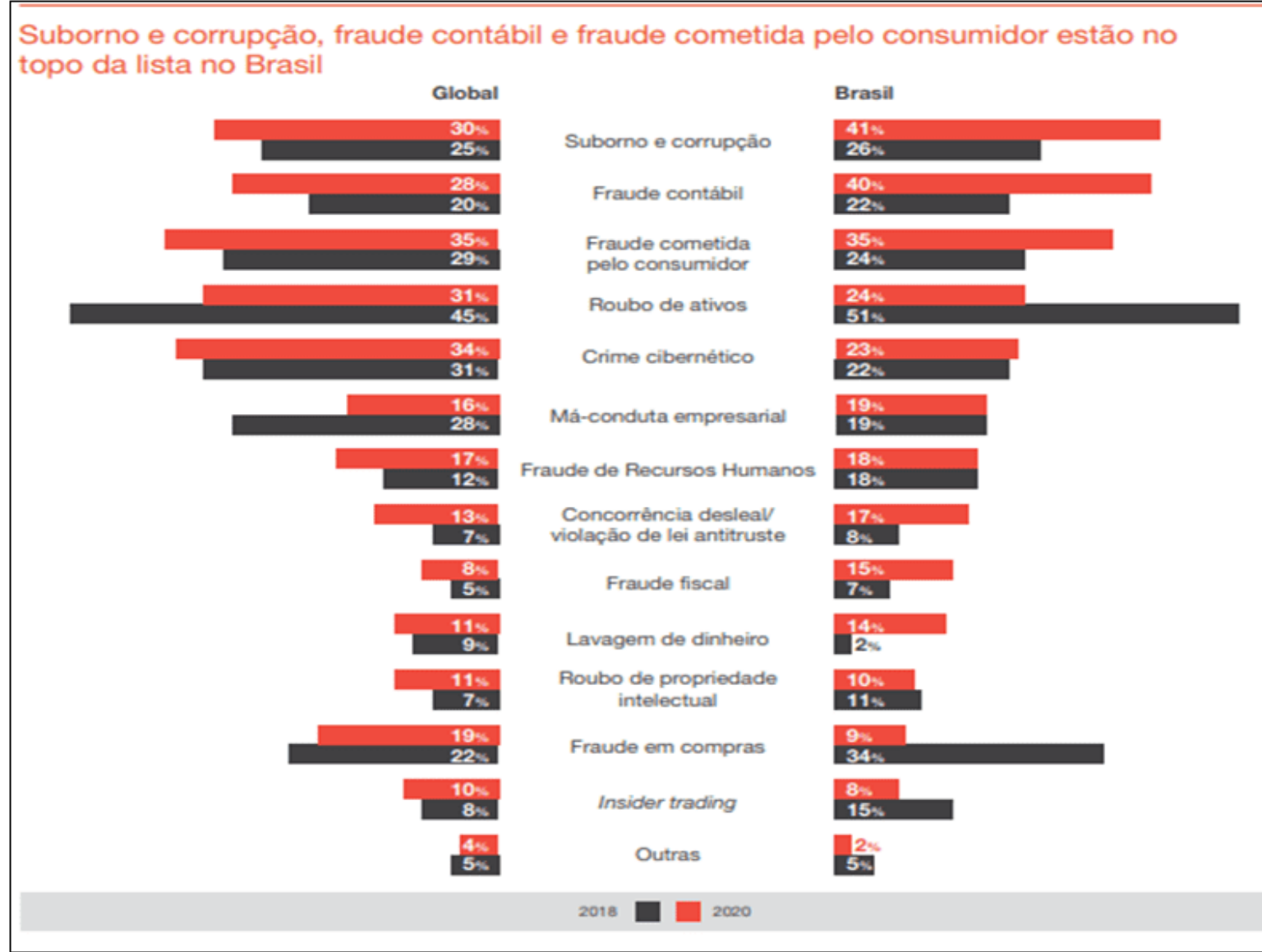

Source: PwC Brasil (2020, p. 5)

Ce sont des crimes économiques qui, en plus d'endommager les actifs des organisations, compromettent leur réputation et leur image, étant possibles jusqu'à ce qu'ils atteignent la faillite. C'est dans cette perspective que les programmes de conformité permettent d'éviter les risques, les comportements illicites et contraires à l'éthique, dès la mise en œuvre d'un programme spécifique qui contient clairement le Code d'éthique et la politique interne de l'organisation à suivre, conférant « croissance, investissement, crédibilité, sécurité et protection » (PwC, 2020).

En ce sens, Oliveira (2012 apud COSSENZO, 2015, p. 35) affirme que les soins de prévention devraient être essentiellement composés de l'engagement que les entreprises doivent établir en interne, lié à la conduite éthique des décisions et des

RC: 103917

Disponible en: https://www.nucleodoconhecimento.com.br/administration-desaffaires/avantages-de-la-conformite 
comportements, imprégnant leurs processus commerciaux et opérationnels, et peut même adopter des pratiques préventives, liées à l'histoire des employés, des fournisseurs et des clients pour, renforcer une culture antifraude interne et externe.

Parmi les risques de la conformité, il convient de mentionner la théorie du triangle de la fraude, qui met en évidence trois aspects qui augmentent les risques d'actes illégaux contre les organisations, sont: la pression, l'opportunité et la rationalisation, comme l'expliquent Condé, Almeida et Quintal (2015). Ce triangle peut être vu par la figure 6.

Figure 6: Triangle de la fraude

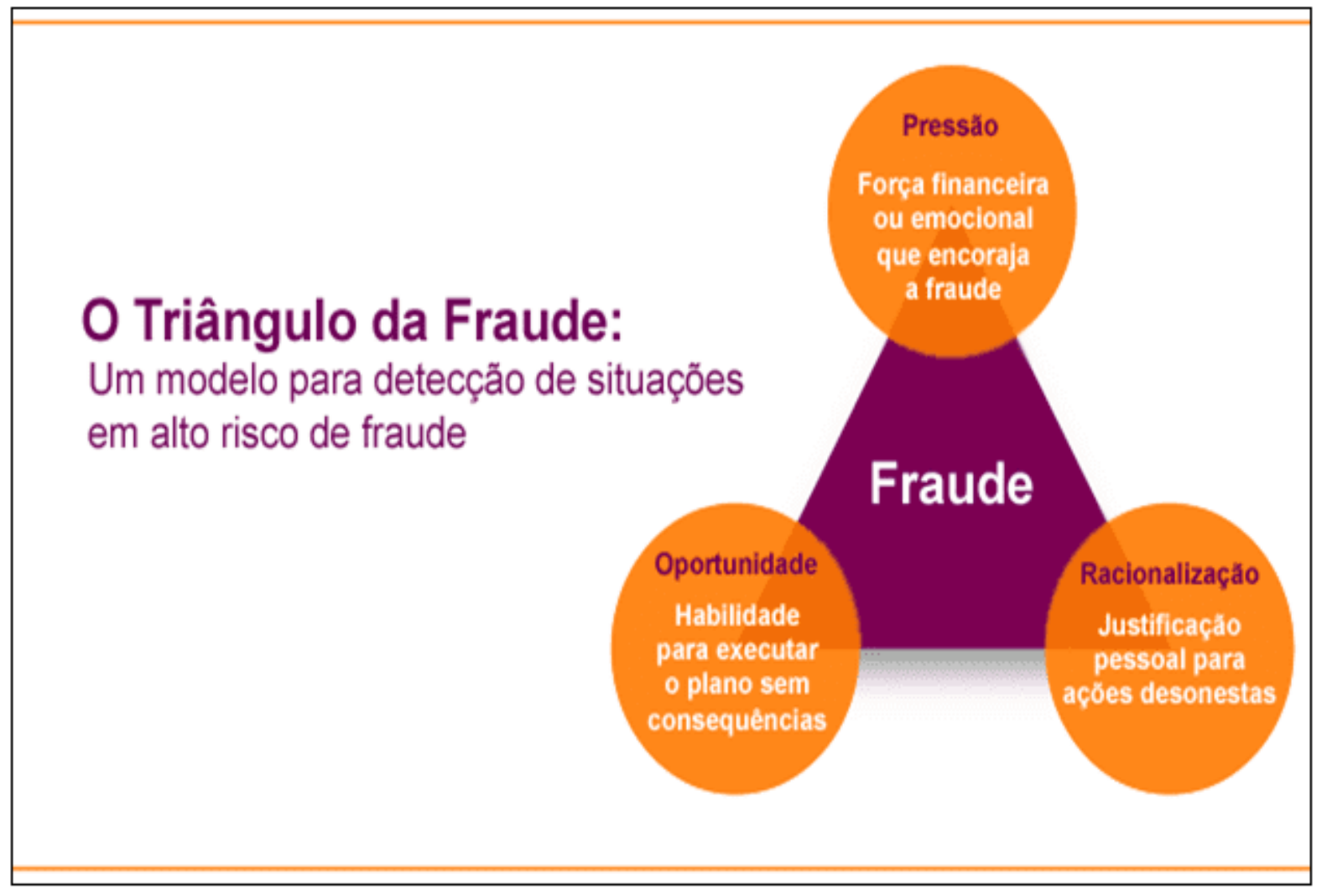

Source: Lustosa (2016)

Toujours Condé et al. (2015) définir chacun des éléments qui composent le triangle de la fraude: 
"oportunidade" de cometer o ato ilícito, com baixo risco de ser descoberto ou nenhum. A "Racionalização" de uma autojustificação pelo cometimento do ilícito que para o fraudador seria "aceitável".

Compte tenu de ce scénario, nous notons la nécessité d'une cartographie des risques organisationnels, à travers la prévention et l'identification des risques, y compris le non-respect de la législation et du code de conduite organisationnel, le risque opérationnel, le risque d'image dû à la négligence ou à l'absence de conduite appropriée, ou même les risques externes tels que la demande politique du pays, les perturbations technologiques, l'instabilité sanitaire et économique, des aspects qui peuvent également affecter les organisations.

En ce sens, Cossenzo (2015) explique que pour gérer la fraude, des stratégies sont nécessaires pour les identifier à l'heure actuelle, en plus d'autres, qui permettent leur prévention. Lorsque les risques de fraude sont identifiés, il est également nécessaire d'identifier comment ils se produisent et les individus qui les pratiquent, et pour cela, il est essentiel que les entreprises partagent des informations entre elles.

Ce même auteur souligne l'existence des Commissions de lutte contre la Fraude de la Fédération Brésilienne des Banques (FEBRABAN), de la Commission de Base Pour l'Incohérence de l'Association Nationale des Institutions de Crédit, de Financement et d'Investissement (ACREFI) et de l'accord de coopération signé en 2009 entre la Police Fédérale et Febraban, en ce qui concerne les contrôles électroniques avec des informations sur les cas qui se sont produits (COSSENZO, 2015, p. 35).

En ce qui concerne la fraude externe, Oliveira (2012, p. 73-4) mentionné par Cossenzo (2015, p. 36) enseigne qu'il existe trois phases préventives: « prévention, détection et réaction », toutes dans une phase globale, appelée «continue ». De telles phases peuvent être observées dans le graphique 1.

Tableau 1 - Phases et catégories de Prévention Externe de la Fraude

\begin{tabular}{|l|l|}
\hline Phase & Catégories connexes \\
\hline Continu & Responsabilité \\
\hline
\end{tabular}

RC: 103917

Disponible en: https://www.nucleodoconhecimento.com.br/administration-desaffaires/avantages-de-la-conformite 


\begin{tabular}{|l|l|}
\hline & Synergie \\
\hline Prévention & Amélioration \\
\hline \multirow{2}{*}{ Détection } & Institution \\
\hline & Identification \\
\hline Réaction & Entretien \\
\hline & Recherche \\
\hline & Sanctions \\
\hline
\end{tabular}

Source: Préparé par l'auteur (2021)

Pour prévenir de tels événements, des mesures devraient être adoptées pour sensibiliser les personnes concernées, et des contrôles devraient être définis et mis en œuvre, empêchant ainsi de nouveaux événements; pour leur détection, il convient d'adopter des méthodes d'enquête et de confirmation des soupçons concernant la survenance ou non d'une fraude; et de réagir, que des actions sont établies par l'entreprise, lorsque la fraude est constatée.

\subsection{SOLUTIONS ENTERPRISE GOVERNANCE, RISK MANAGEMENT AND COMPLIANCE (EGRC)}

Parmi les avantages que la mise en œuvre d'un programme de conformité et de gestion des risques favorise au sein des organisations figurent la protection contre la fraude et l'illicite, la promotion de l'atténuation de ces risques.

Pour que les mesures de sécurité soient efficaces, grâce à une réalité de plus en plus technologique, il est nécessaire que des outils et des solutions soient développés et mis en œuvre pour lutter contre la fraude, permettant une plus grande conformité aux pratiques nécessaires au succès des organisations, comme l'explique Grand View Research (2014). Ce sont des mesures inhérentes à la gouvernance des technologies de l'information, ainsi qu'à l'Internet of things (IoT), ou à l'Internet des objets, qui à leur tour sont inhérentes à la Gouvernance d'Entreprise (CHAVES, 2014).

RC: 103917

Disponible en: https://www.nucleodoconhecimento.com.br/administration-desaffaires/avantages-de-la-conformite 
La gouvernance d'entreprise consiste en un « système de relations entre actionnaires, auditeurs indépendants, dirigeants d'entreprise et administrateurs dirigé par le conseil d'administration » (LODI, 2000, mentionné par CHAVES, 2014, p. 21). À son tour, la Gouvernance Informatique, par le biais du « Big Data et de l'analyse, de l'loT, de l'Apprentissage Automatique, de l'IA et des médias sociaux, implique le partage d'informations personnelles, rendant les utilisateurs et les entreprises plus vulnérables aux pirates informatiques » (GRAND VIEW RESEARCH, 2014).

Les ressources apportées par l'loT et la numérisation des processus métier se sont développées rapidement, permettant des interactions de données et offrant un meilleur contrôle de l'information par la direction des entreprises. En pratique, l'objectif est de s'appuyer sur l'Intelligence Artificielle (IA) pour gérer les obligations et les rapports externes, ce qui permet une meilleure gouvernance d'entreprise, une meilleure gestion des risques et de la conformité - EGRC (GRAND VIEW RESEARCH, 2014).

Selon Chaves (2014, p.22), « pour l'Institut Brésilien de Gouvernance d'Entreprise (IBGC, 2006), la Gouvernance Informatique est une branche de la gouvernance d'entreprise », ce qui permet l'élaboration et la mise en œuvre de « réglementations à fort impact dans le domaine informatique des entreprises, nécessitant un engagement et une efficacité accrus dans la planification, les contrôles, la surveillance et les processus de sécurité liés aux stratégies commerciales ». Toujours Chaves (2014, p. 22) rapporte une définition donnée par I'Information Technology Governance Institute, (ITGI, 2012), en ce qui concerne les objectifs de la gouvernance informatique:

compreender os assuntos e a importância estratégica de TI, assegurar que a empresa possa sustentar suas operações e implementar as estratégias requeridas para expandir as suas atividades no futuro. As práticas de Governança de TI possibilitam que: sejam atendidas as expectativas dos clientes de TI, que a performance de TI seja medida, os seus recursos possam ser gerenciáveis e os riscos do negócio mitigados.

À son tour. Siqueira (2019) fait référence à l'enquête sur la cybersécurité et les risques liés aux données, préparée par Marsh et McLennan en 2018, démontrant que 
seulement $18 \%$ des petites entreprises auraient élaboré une sorte de plan d'intervention en cas de cyberincident.

Dans cette perspective, la création et la mise en œuvre de solutions EGRC offrent la possibilité de surmonter les défis existants - tels que la fraude électronique et le clonage, par exemple, dans un univers commercial « hyperconnecté », qui peut être obtenu via un logiciel spécifique. II représente également un outil qui collabore substantiellement dans le domaine de la protection des données et de la Propriété Intellectuelle $(\mathrm{PI})$, en plus d'améliorer la gestion du travail à distance, un modèle hybride si commun tout au long de la pandémie de Covid 19 (GRANDVIEW RESEARCH, 2014).

\section{AVANTAgES D'UN PROGRAMME DE CONFORMITÉ ET DE GESTION DES RISQUES}

Selon le portail 3Mind Legal (2021), parmi les principaux concepts de conformité et degestion des risques, figure la génération de valeur commerciale, qui vise à permettre la survie et la longévité des organisations. Parmi les dangers et les impacts financiers que l'absence d'un programme qui favorise la bonne gouvernance sont: « l'absence de lignes directrices normatives; le manque d'outils de prévention adéquats; les désalignements avec les lois applicables; système d'information sans opérations structurées; la gestion des processus défaillants.

Étant donné que les institutions publiques et privées devraient guider leur gestion dans les programmes de Gouvernance d'Entreprise, Klen (2009, apud SENNO et al., 2019, p. 225) définit que « la gouvernance d'entreprise est le système par lequel les sociétés commerciales sont gérées et contrôlées ».

En ce sens, toujours Senno et al. (2019) expliquent que les pratiques en matière de Gouvernement d'Entreprise doivent converger pour garantir des mécanismes permettant le rendement financier des investissements réalisés, grâce à l'adoption de règles juridiques et à la responsabilité vis-à-vis de toutes les parties prenantes.

RC: 103917

Disponible en: https://www.nucleodoconhecimento.com.br/administration-desaffaires/avantages-de-la-conformite 
À leur tour, Azevedo et al. (2017, p. 181) expliquent que la gouvernance se réfère au gouvernement, tandis que la gouvernance d'entreprise se réfère au système par lequel les organes et les pouvoirs sont organisés au sein d'une entreprise ", qui doit être soutenu par quatre principes: transparence, intégrité ou équité, responsabilité et respect des lois.

Ces explications visent à collaborer pour mieux comprendre les bonnes pratiques de gouvernance qui, selon KPMG (2021), nécessitent certains éléments spécifiques pour constituer un programme de conformité réglementaire efficace, à savoir:

1. Politique et programme de conformité;

2. Message de leadership et culture de conformité;

3. Structuration du responsable de la conformité;

4. Communication et formation de la politique et du programme de conformité;

5. Surveillance, vérification et évaluation de l'efficacité de la politique et du programme d'éthique et de conformité;

6. Mesures incitatives en faveur du rendement et mesures disciplinaires;

7. Recherche et prévention.

Dans cette perspective, il est signalé qu'un programme de conformité implique des normes de lutte contre la corruption, de corruption et de conformité publique. Pour plus de détails, les avantages fournis par Compliance sont énumérés,selon le portail 3Mind Legal (2021):

1. Attirer les investisseurs et les investissements;

2. Augmenter la gouvernance dans l'entreprise;

3. Consolider la culture organisationnelle;

4. Corriger efficacement les non-conformités;

5. Gagner en crédibilité;

6. Obtenir un avantage concurrentiel sur la concurrence;

7. Identifier les risques et prévenir les problèmes;

8. Améliorer l'efficacité et la qualité des produits et services offerts; 
9. Durabilité.

À son tour, Caovilla (2017) décrit certains avantages liés à l'adoption de la conformité par les organisations (nos griffons):

1. Nouvelles opportunités commerciales et avantage concurrentiel: les entreprises qui opèrent conformément aux lois et aux bonnes pratiques cherchent à établir des relations égales, en plus d'accroître leurs opportunités par rapport à celles qui ne sont pas des utilisateurs de programmes de conformité;

2. Nouveaux investissements: les organisations peu impliquées dans des scandales illicites et sociaux, faisant preuve de solidité, sont plus attrayantes pour les investisseurs internes et externes;

3. Atténuation des risques: les entreprises qui adoptent un programme de conformité, en plus d'identifier les risques possibles pour leurs activités, sont en mesure d'élaborer et de mettre en œuvre des mesures pour atténuer les défaillances et les risques possibles;

4. Correction des non-conformités: en plus des actions préventives pour éviter d'éventuelles non-conformités, sont les actions pour corriger les nonconformités identifiées dans les processus internes d'une organisation. De telles actions nécessaires pour préserver l'image et la réputation d'une entreprise peuvent être: correction du plan d'action en cours; la formation des employés; l'examen des politiques, des procédures, de la conduite, des employés, des fournisseurs et des fournisseurs de services;

5. La conformité en tant que sensibilisation des personnes: une pratique qui permet d'atténuer la fraude, car si les employés et les autres acteurs sont formés à la conformité, ilssont en mesure de voir les non-conformités à l'intérieur et à l'extérieur de l'entreprise, c'est-à-dire dans la concurrence, chez les fournisseurs, les prestataires de services;

6. La conformité comme limitation de responsabilité: les connaissances acquises dans la formation et les pratiques éthiques liées à la conformité permettent de sélectionner de meilleurs partenaires commerciaux, en plus de

RC: 103917

Disponible en: https://www.nucleodoconhecimento.com.br/administration-desaffaires/avantages-de-la-conformite 
protéger la réputation d'une entreprise. Étant donné que les bonnes pratiques imprègnent les routines commerciales d'une organisation, toute non-conformité peut entraîner la réduction des pénalités qui peuvent lui être appliquées;

7. La conformité en tant que durabilité commerciale: l'adoption et le maintien de pratiques éthiques et transparentes permettent aux organisations de rester à long terme.

\section{CONCLUSION}

Tout au long de l'analyse des références théoriques sélectionnées pour l'élaboration de cet article, il a été possible d'observer qu'un programme de conformité et de gestion des risques revêt une grande importance pour la bonne gestion des processus, des pratiques et des routines au sein des organisations, impliquant tous leurs secteurs et leur personnel.

Retour au point des rênes; Comment les avantages offerts par le programme de conformité et de gestion des risques collaborent-ils pour réduire les risques de fraude, de criminalité illicite et de corruption au sein des organisations et institutions gouvernementales? Nous concluons que, puisque la littérature démontre qu'il y a des fraudes dans toutes les organisations, sans distinction pour le segment d'activité ou sa taille, l'avantage que la conformité confère aux entreprises réside précisément dans la protection qu'elle offre, en atténuant les différents types de risques et illicites, y compris en évitant les sanctions légales.

Sur la base des lois instituées, de la Loi Pionnière pour Lutter Contre la Corruption FCPA (Foreign Corrupt Practices Act), lespays signataires de l'OCDE, énumérés dans cet article, ont adopté des initiatives similaires pour promouvoir l'atténuation de la fraude, de la corruption et des actions contraires à l'éthique dans leurs gouvernements et organisations, fortement soutenus par les restrictions et les sanctions apportées par les lois. 
En outre, le développement de nouveaux instruments technologiques, appuyés par la gouvernance des technologies de l'information, a émergé pour soutenir la gouvernance d'entreprise, favorisant des changements dans la gestion des organisations et de leurs processus administratifs.

Une telle gestion est quelque chose qui devrait être basé sur des outils modernes, ne se limitant pas aux processus eux-mêmes, mais qui devrait permettre à une organisation d'anticiper les événements futurs, en évitant les malentendus.

Dans ce contexte, les solutions EGRC - qui permettent les meilleures pratiques en matière de gouvernance informatique et de gouvernance d'entreprise - collaborent pour la prévention et l'atténuation de la fraude et des actions non averties, protégeant ainsi la croissance des organisations et leur image de marché.

\section{RÉFÉRENCES}

AZEVEDO, Mateus Miranda de; CARDOSO, Antonio Almeida; DUARTE, Jairo Gonçalves; FEDERICO, Bianca Ellen; LIMA, Marco Antonio Ferreira. O Compliance e a gestão de riscos nos processos organizacionais. Revista de Pós-Graduação Multidisciplinar, São Paulo, v. 1, n. 1, p. 179-196, mar./jun. 2017.

ISSN 2594-4800 | e-ISSN 2594-4797 | doi: 10.22287/rpgm.v1i1.507; acesso em 08 dez 2021.

CAOVILLA, Renato Vieira. Benefícios práticos do compliance. 10 jul 2017. Disponível em: [https://www.migalhas.com.br/depeso/261662/beneficios-praticos-docompliance]; 08 dez 2017.

CHAVES, Elisabete Cecília Januário. Resultados e tendências de Requisitos e Práticas de Governança de TI nos bancos de Varejo no Brasil. Dissertação [Mestrado em Gestão e Tecnologia em Sistemas Produtivos] apresentada ao Centro Estadual de Educação Tecnológica Paula Souza, no Programa de Mestrado Profissional em Gestão e Tecnologia em Sistemas Produtivos. São Paulo, novembro

RC: 103917

Disponible en: https://www.nucleodoconhecimento.com.br/administration-desaffaires/avantages-de-la-conformite 
de

2014.

Dsponível

em:

[http://www.pos.cps.sp.gov.br/files/dissertacoes/file/143/7fc218f434af24b99f50af42e 8442903.pdf]; acesso em 12 out 2021.

COELHO, Cláudio Carneiro Bezerra Pinto; SANTOS JÚNIOR, Milton de Castro. Compliance, FGV/IDE, 2021.

CONDÉ, Robson Augusto Dainez; ALMEIDA, Carlos Otávio Ferreira de; QUINTAL, Renato Santiago. Fraude Contábil: análise empírica à luz dos pressupostos teóricos do triângulo da fraude e dos escândalos corporativos. Gestão \& Regionalidade - Vol. 31 - № 93 - set-dez/2015. doi: 10.13037/gr. vol31n93.2929; acesso em 28 out 2021

COSSENZO, Fábio. FRAUDE BANCÁRIA: A mitigação dos Riscos e Perdas Financeiras no Segmento de Crédito Consignado. Monografia de Pós-Graduação Lato Sensu - MBA Gestão empresarial, apresentada à Fundação Instituto de Administração - FIA. São Paulo, 2015.

DURÃES, Cintya Nishimura; RIBEIRO, Maria de Fátima. O Compliance no Brasil e a Responsabilidade Empresarial no combate à corrupção. Revista Direito em Debate: Revista do Departamento de Ciências Jurídicas e Sociais da Unijuí Editora Unijuí - Ano XXIX - n. 53 - jan./jun. 2020. Disponível em: [http://dx.doi.org/10.21527/2176-6622.2020.53.69-78]; acesso em 01 dez 2021.

ENTERPRISE GOVERNANCE, RISK \& COMPLIANCE MARKET SIZE. Share \& Trends Analysis Report By Component, By Software, By Services, By Enterprise Type, By Vertical, And Segment Forecasts, 2021 - 2028

FGV - FUNDAÇÃO GETÚLIO VARGAS. Surgimento do Compliance. 2021.

GONSALES, Alessandra. O que é compliance? Publicação da Legal, Ethics and Complaince (LEC). Vídeo publicado em 29 fev 2016. Disponível em: [https://www.youtube.com/watch?v=2BDpJ6UMXb4]; acesso em 01 dez 2021. 
GRAND VIEW RESEARCH. Relatório de governança corporativa, risco e conformidade do mercado, análise de compartilhamento e tendências por componente, por software, por serviços, por tipo de empresa, por vertical e por segmento. Previsões, 2021-2028. Artigo publicado em abril de 2021. Disponível em: [https://www.grandviewresearch.com/industry-analysis/enterprise-governance-riskcompliance-egrc-market]; acesso em 20 nov 2021.

HAUSSEN, Leonard. Compliance. Reunião On-Line (ROL), Aula Organizada pela Fundação Getúlio Vargas, Escola de Administração de Empresas de São Paulo, São Paulo, Brasil. Agosto de 2021.

JORGE, Glaucio Fiorenzano; TOMAZ, Roberto Epifânio. Compliance rins - como implantar e quais os benefícios do programa de compliance? IV Congresso Catarinense de Direito processual Civil \& Mais. 7 a 9 de novembro de 2018. Univali Eventos - Universidade Vale do Itajaí. Disponível em: [https://core.ac.uk/download/pdf/276005736.pdf]; acesso em 20 out 2021.

KPMG - Compliance analytics. 2018. Disponível em: [https://assets.kpmg/content/dam/kpmg/br/pdf/2018/08/br-compliance-analytics.pdf]; acesso em 08 dez 2021.

KPMG - Compliance regulatório. 2021. Disponível em: [https://home.kpmg/br/pt/home/servicos/consultoria/risk-consulting/internal-auditrisk/compliance-regulatory.html]; acesso em 08 dez 2021.

MELO, Luísa. 8 crises empresariais causadas por funcionários brincalhões. Matéria publicada em 14 mar 2014. Disponível em: [https://exame.com/negocios/8crises-empresariais-causadas-por-funcionarios-brincalhoes/]; acesso em 20 out 2021.

MICHELOTTI, Andriws Loreto. O crime de lavagem de capitais e os desafios empresariais diante da implementação dos programas de criminal Compliance. Trabalho de Conclusão de Curso [Bacharelado em Direito] apresentado à Faculdade Antonio Meneghetti (AMF). Restinga Seca (RS), 2020. Disponível em: 
[http://repositorio.faculdadeam.edu.br/xmlui/bitstream/handle/123456789/673/TCC_D IR_ANDRIWS_MICHELOTTI_AMF_2020.pdf?sequence=1\&isAllowed $=y$ ]; acesso em 07 dez 2021.

NASCIMENTO, Juliana Oliveira. A Função Sociald da Empresa e a Efetividade Prática da Conduta de Integridade: o Compliance Vivo. Dissertação [mestrado em Direitos Fundamentais e Democracia] apresentada ao Centro Universitário Autônomo do Brasil. Curitiba, 2018. Disponível em: [https://www.unibrasil.com.br/wpcontent/uploads/2019/07/Dissertação-JULIANA-OLIVEIRA-NASCIMENTO.pdf]; acesso em 29 ago 2021.

NASCIMENTO, Débora Minuncio. Evolução histórica e legislações acerca do Compliance. Artigo publicado em 07 nov 2021. Disponível em: [https://advocaciadeboramn.jusbrasil.com.br/artigos/700763578/evolucao-historica-elegislacoes-acerca-do-compliance]; acesso em 20 out 2021.

\section{OECE - ORGANIZAÇÃO PARA A COOPERAÇÃO E DESENVOLVIMENTO ECONÔMICO INTERNACIONAL. \\ Disponível \\ em:}

[https://www.dgae.gov.pt/servicos/comercio-internacional-e-relacoesinternacionais/multilaterais/organizacao-para-a-cooperacao-e-desenvolvimentoeconomico-ocde-.aspx]; acesso em 08 dez 2021.

PwC. Combate a fraudes e crimes econômicos: uma batalha sem fim Pesquisa Global sobre Fraudes e Crimes Econômicos 2020. Disponível em: [https://www.pwc.com.br/pt/publicacoes/servicos/assets/consultorianegocios/2020/pesquisa_sobre-fraudes-e-crimes-economicos-2020-pwc-brasil.pdf]; acesso em 20 out 2021.

SENNO, Elisângela Pereira; PRATES, Glaucia Aparecida; LUCENTE, Adriano dos Reis; GALLI, Lesley Carina do Lago Attadia; GALLI, Rafael Altafin. Benefícios da implantação do programa de Compliance em uma organização do setor de energia: um estudo de caso. II SITEFA - Simpósio de Tecnologia Fatec Sert5aozinho. Disponível em: 
[https://sitefa.fatecsertaozinho.edu.br/index.php/sitefa/article/view/90/51]; acesso em 08 dez 2021.

SIQUEIRA, Marina Paiva de. Cyber Risks: Fatores decisórios para a transferência mediante a contratação do seguro. Monografia [Bacharelado em Administração] apresentada ao Departamento de Administração da Universidade Federal Fluminense. Niterói, RJ. 2019.

SOBREIRA FILHO, Enoque Feitosa; LEITE, Flavia Piva Almeida; MARTINS, José Alberto Monteiro. Ética empresarial como base de sustentação do programa de Compliance: uma breve análise sobre a ética, a integridade e o Compliance. Revista Relações Internacionais no Mundo Atual. Vol. 2,n. 23, 2019. Disponível em: [http://revista.unicuritiba.edu.br/index.php/RIMA/article/view/3891/371373033]; acesso em 29 ago 2021.

SOUZA, Silvia Regina; MACIEL-LIMA, Sandra; LUPI, André Lipp Pinto Basto. Aplicabilidade do Compliance na Administração Pública em face ao momento político atual brasileiro. Percurso - Anais do I Congresso Ibro-Americano de Direito Empresarial e Cidadania (CONIBADEC), vol. 01, n. 24, Curitiba, p.1-22, 2018. Disponível em: [http://revista.unicuritiba.edu.br/index.php/percurso/article/view/3274/371371782]; acesso em 01 dez 2021.

3MIND JURÍDICO. Compliance: o que é e o benefício para empresas. Abril de 2021. Disponível em: [https://www.3mind.com.br/blog/compliance-empresas/]; acesso em 08 dez 2021.

VAZ, Tatiana. 5 empresas envolvidas em escândalos recentes. Artigo publicado em 30 abr 2012. Disponível em: [https://exame.com/negocios/5-empresas-envolvidasem-escandalos-recentes/]; acesso em 28 out 2021. 
VEJA ON LINE. Funcionário de restaurante publica foto lambendo comida de clientes. Junho de 2013.2 Disponível em: [https://veja.abril.com.br/economia/funcionario-de-restaurante-publica-fotolambendo-comida-de-clientes/]; acesso em 14 out 2021.

Soumis: Novembre, 2021.

Approbation: Décembre 2021. 